\title{
Labordiagnostik der Leberfibrose und der nichtalkoholischen Fettleber-Krankheit
}

\author{
von Eckardstein, Arnold
}

\begin{abstract}
Durch ihre hohe Prävalenz und die assoziierten Folge-Erkrankungen sind Leberfibrose jeglicher Ätiologie sowie die nicht-alkoholische Fettlebererkrankung (NAFLD) wichtige öffentliche Gesundheitsprobleme. Die Leberbiopsie gilt als Goldstandard für die Diagnostik und die Stadieneinteilung der Leberfibrose und NAFLD. In den letzten Jahren wurden etliche auf Laborparameter und/oder Bildgebungsverfahren basierende nichtinvasive Verfahren für die Diagnostik von Leberfibrose bei chronischer Hepatitis und NAFLD entwickelt und validiert. Im Vergleich zu den patentierten Tests (FibroTest
\end{abstract}

DOI: https://doi.org/10.1515/labmed-2013-0054

Other titles: Laboratory diagnostics of liver fibrosis and non-alcoholic fatty liver disease

Posted at the Zurich Open Repository and Archive, University of Zurich

ZORA URL: https://doi.org/10.5167/uzh-153920

Journal Article

Published Version

Originally published at:

von Eckardstein, Arnold (2014). Labordiagnostik der Leberfibrose und der nichtalkoholischen FettleberKrankheit. LaboratoriumsMedizin, 38(2):75-85.

DOI: https://doi.org/10.1515/labmed-2013-0054 


\title{
Klinische Chemie und Stoffwechsel/ Clinical Chemistry and Metabolism
}

Redaktion: W. März

Arnold von Eckardstein*

\section{Labordiagnostik der Leberfibrose und der nichtalkoholischen Fettleber-Krankheit ${ }^{1)}$}

\author{
Laboratory diagnostics of liver fibrosis and non-alcoholic fatty liver disease
}

Zusammenfassung: Durch ihre hohe Prävalenz und die assoziierten Folge-Erkrankungen sind Leberfibrose jeglicher Ätiologie sowie die nicht-alkoholische Fettlebererkrankung (NAFLD) wichtige öffentliche Gesundheitsprobleme. Die Leberbiopsie gilt als Goldstandard für die Diagnostik und die Stadieneinteilung der Leberfibrose und NAFLD. In den letzten Jahren wurden etliche auf Laborparameter und/oder Bildgebungsverfahren basierende nichtinvasive Verfahren für die Diagnostik von Leberfibrose bei chronischer Hepatitis und NAFLD entwickelt und validiert. Im Vergleich $\mathrm{zu}$ den patentierten Tests (FibroTest ${ }^{\circledR}$, Fibrometer ${ }^{\circledR}$ und Hepascore ${ }^{\circledR}$ ) haben die nicht-patentierten Tests (APRI, ELFG, FIB-4, Forns Index und MP3) eine tendenziell geringere diagnostische Qualität, insbesondere bei der Diagnose einer signifikanten Fibrose (METAVIR- Stadium F2). Der Qualitätsunterschied ist weniger ausgeprägt für die Diagnose einer Zirrhose (METAVIR-Stadium F4). Die Elastographie ist den Biomarkern bei der Diagnose einer Zirrhose (F4) überlegen, nicht aber bei der Diagnose einer Fibrose (F2). Allerdings schränkt die Nichtdurchführbarkeit der Elastographie bei ca. 20\% der Patienten und die eingeschränkte Verfügbarkeit des Systems die klinische Anwendung ein. Cytokeratin 18 (CK-18) ist der derzeit meistversprechende Einzelparameter für die Diagnose der nicht-alkoholischen Fettleberhepatitis (NASH). Die Datenlage zur Wertigkeit der Scores und Algorithmen zur Diagnostik und Schweregrad-Einteilung von NAFLD und NASH ist im Vergleich zur Wertigkeit der Scores für die chronische Hepatitis weniger gut abgesichert aber vielversprechend. Die patentierten Scores und CK-18 scheinen den freiverfügbaren Scores inkl. des von den Guidelines favorisierten NAFLD-Fibrose Scores überlegen. Zusammengefasst erscheinen die neuen nicht-invasiven Verfahren insbesondere als Prä-Screening-Verfahren geeignet, die Zahl der Leberbiopsien zu begrenzen.
Schlüsselwörter: chronische Hepatitis; Fettlebererkrankung; Leberfibrose.

Abstract: Owing to the high prevalence and associated complications of liver fibrosis, of any etiology, and nonalcoholic fatty liver disease (NAFLD), both have become important public health issues. Liver biopsy is considered the gold standard for diagnosis and staging of liver fibrosis, as well as NAFLD. Recent studies have discovered and validated several non-invasive biochemical biomarkers and imaging procedures for the diagnostics of liver fibrosis and NAFLD. In comparison to patented tests (FibroTest ${ }^{\circledR}$, Fibrometer $^{\circledR}$, and Hepascore ${ }^{\circledR}$ ), non-patented tests (APRI, ELFG, FIB-4, Forns Index, and MP3) tend to have a lower diagnostic performance, especially for the diagnosis of significant fibrosis (METAVIR stage F2). The difference in performance is less pronounced for the diagnosis of cirrhosis (METAVIR stage F4). Elastography is superior to biomarkers in the diagnosis of cirrhosis (F4) but not fibrosis (F2). However, in 20\% of patients elastography cannot be performed or evaluated due to anatomical reasons. Cytokeratin 18 (CK-18) is the most promising single biomarker for the diagnosis of non-alcoholic steatohepatitis (NASH). Scores and algorithms have been less extensively validated for their diagnostic performance in diagnosing and staging of NAFLD and NASH as compared with fibrosis in chronic hepatitis. Data are promising. Patented scores as well as CK-18 appear slightly superior to freely available scores including the NAFLD fibrosis score, which is recommended by American guidelines. In conclusion, noninvasive biomarkers and elastography appear promising as prescreening tools to limit the number of liver biopsies.

Keywords: chronic hepatitis; fatty liver diseases; liver fibrosis.

1) Dieser Artikel beruht auf einem Skript zum Diagnostik-Update 2013 (08.-09. März 2013, Mannheim). 
*Korrespondenz: Arnold von Eckardstein, Institut für Klinische Chemie, Universitätsspital Zürich, Rämistrasse 100, 8091 Zürich, Schweiz, Phone: +0041 44255 2260, Fax: +0041 4425545 90, E-Mail: arnold.voneckardstein@usz.ch

\section{Einleitung}

Lebererkrankungen sind durch vier grundsätzliche Pathomechanismen gekennzeichnet, von denen drei durch klassische labormedizinische Parameter erfasst werden: Nekrose, Cholestase und Metabolische Insuffizienz. Die Fibrose und ihre ausgeprägteste Form, die Zirrhose, haben sich hingegen lange Zeit der nicht-invasiven Diagnostik verschlossen. Mit dem Fortschritt der therapeutischen Möglichkeiten ist der Bedarf für eine zuverlässige Diagnostik der Leberfibrose massiv gestiegen. Allerdings ist die Entwicklung von Biomarkern für das nicht-invasive Staging der Leberfibrose schwierig, weil sie eine Komponente des normalen Heilungsprozess nach Verletzungen, Infektionen und vielen anderen ätiologischen Faktoren darstellt. Seit Jahrzehnten gilt die Leberbiopsie als Goldstandard für die Diagnostik und die Stadieneinteilung der Leberfibrose, zumal sie auch die Beurteilung von Nekrose, Entzündungsaktivität sowie Einlagerungen von Fett (Steatose), Eisen oder Kupfer ermöglicht. In den letzten Jahren wurden etliche auf Laborparametern und/ oder Bildgebungsverfahren basierende indirekte Verfahren entwickelt und validiert, die allerdings international sehr unterschiedlich in die klinische Praxis übernommen wurden. Die nicht-invasiven Verfahren scheinen insbesondere als Prä-Screening-Verfahren geeignet, um die Zahl der Leberbiopsien zu begrenzen [1-4].

\section{Goldstandard Leberbiopsie}

Obwohl Goldstandard hat die Leberbiopsie erhebliche Nachteile wie begrenzte Zugänglichkeit, hohe Kosten und Patientengefährdung. Unter den Komplikationen sind Schmerz (85\%) und Blutdruckabfall die häufigsten, intraperitoneale Blutung (ca. 0,2\%) und Verletzung die schwerwiegendsten. Das Risiko für Hospitalisierung nach Leberbiopsie beträgt 1 bis 5 Prozent, die Mortalität ca. 0,01\%. Aus Angst vor diesen Komplikationen vermeiden viele Patienten eine Leberbiopsie. Zudem ist die Qualität der Leberbiopsie durch Fehler bei der Probensammlung und -beurteilung (Unrichtigkeit) sowie Reproduzierbarkeit (hohe intra- und interobserver-Variabilität) eingeschränkt [4].

Die am häufigsten eingesetzten Methoden zum semiquantitativen Scoring des Fibrosegrades sind der Ishak- und der Metavir-Score, die ursprünglich zur Beurteilung der chronischen Hepatitis entwickelt wurden, sowie der Brunt-Score, der für die Beurteilung der nichtalkoholischen Fettleber entwickelt wurde (Tabelle 1). Der wichtigste die Qualität limitierende Faktor dieser standardisierten Methoden ist die Probengewinnung, welche bei einem Drittel der Fälle zu fehlerhaften oder zwischen verschiedenen Pathologen diskrepanten Stadieneinteilungen führt. Entsprechend sollen Biopsien mindestens 20-25 mm lang sein und/oder mindestens 11 Portalfelder enthalten. Leider wird diese Vorgabe nur von etwa der Hälfte der Biopsien erfüllt. Eine andere Limitation ist die nichtlineare Beziehung zwischen Fibrosegrad und klinischer Relevanz. So ist der F0 $\rightarrow$ F1-Übergang klinisch weniger relevant als der F2 $\rightarrow$ F3-Übergang. Für Zirrhose (F4) gibt es keine Sub-Differenzierung des

Tabelle 1 Semiquantitative histologische Methoden zur Beurteilung der Leberfibrose [4].

\begin{tabular}{|c|c|c|}
\hline \multicolumn{2}{|l|}{ Chronische Hepatitis B oder C } & \multirow{2}{*}{$\frac{\text { NAFLD }}{\text { Brunt }}$} \\
\hline METAVIR & Ishak & \\
\hline F0=keine Fibrose & F0=keine Fibrose & F0=keine Fibrose \\
\hline $\mathrm{F} 1=$ portale & F1=Bindegewebsvermehrung einiger Portalzonen mit oder ohne & $\mathrm{F} 1 \mathrm{~A}=$ Milde perisinusoidale Fibrose \\
\hline Bindegewebsvermehrung & fibrösen Septen & \\
\hline $\mathrm{F} 2=$ Periportale Fibrose mit & F2 $=$ Bindegewebsvermehrung der meisten Portalzonen mit oder & $\mathrm{F} 1 \mathrm{~B}=$ Moderate perisinusoidale \\
\hline wenigen Septen & ohne fibrösen Septen & Fibrose \\
\hline F3=Ausgedehnte & F3=Bindegewebsvermehrung der meisten Portalzonen mit & F1C=ausschließliche portale/ \\
\hline Brückenbildung & gelegentlicher portaler Brückenbildung & periportale Fibrose \\
\hline \multirow{5}{*}{$\mathrm{F} 4=$ Zirrhose } & F4=Bindegewebsvermehrung der meisten Portalzonen & F2=Sowohl perisinusoidale als \\
\hline & mit ausgedehnten portal-portaler und portal-zentraler & auch portale/periportale Fibrose \\
\hline & Brückenbildung & \\
\hline & $\begin{array}{l}\text { F5=Ausgedehnte Brückenbildung mit gelegentlichen Knoten } \\
\text { (inkomplette Zirrhose) }\end{array}$ & F3=Brückenbildende Fibrose \\
\hline & F6=Zirrhose & F4=Zirrhose \\
\hline
\end{tabular}


Schweregrades [4]. Zusammengenommen verdeutlichen diese Einschränkungen, dass der Goldstandard Leberbiopsie nicht ideal ist.

Nicht-invasive Methoden haben mehrere grundsätzliche Vorteile gegenüber der Leberbiopsie. Im Vergleich zu dieser sind sie nahezu frei von Nebenwirkungen, präanalytisch leichter beherrschbar, objektiver weil weniger abhängig vom Untersucher, leicht wiederholbar und kostengünstiger. Allerdings zeigen sie nur eine begrenzte Übereinstimmung mit der Histologie. Zudem sind sie stark von der spezifischen Ätiologie der zugrundeliegenden Lebererkrankung sowie anderen klinischen Randbedingungen des Patienten (z. B. Medikamenten) beeinflusst.

Die eingeschränkte Qualität und Zuverlässigkeit des Goldstandards Leberbiopsie führt dazu, dass in vergleichenden Querschnittsstudien Biomarker per definitionem der Leberbiopsie in ihrer diagnostischen Aussagekraft unterlegen sind. Dieses Dilemma kann nur durch Längsschnittstudien gelöst werden, in denen der prädiktive Wert von Biopsien und Biomarkern in Bezug zu klinischen Endpunkten, z. B. Tod, portale Hypertension oder Pro-/Regression der Fibrose in Reaktion auf Alkoholabstinenz, virostatische Therapie bei Hepatitis B und C oder verbesserter Stoffwechsel-Situation bei NASH, verglichen werden.

\section{Im Blut messbare Biomarker}

In den vergangenen Jahren wurden etliche mögliche Biomarker der Leberfibrose entdeckt und in oft kleinen Studien evaluiert. Prinzipiell lassen sich die Blutmarker in direkte und indirekte Marker differenzieren: Direkte Marker sind Moleküle, welche aus der extrazellulären Matrix oder aus Sternzellen freigesetzt werden, z. B. Hyaluronsäure oder $\alpha 2$-Makroglobulin. Indirekte Marker sind Moleküle, welche die Reaktion des Leberparenchyms auf die Fibrose widerspiegeln: Zelltod (ALT), Cholestase (Bilirubin, $\gamma \mathrm{GT}$ ), metabolische Insuffizienz (INR), und portale Hypertension (Thrombozytopenie, Hypergammaglobulinämie). Direkte Marker zeigen die Fibrose zwar früher an, sind aber unspezifischer, weil sie auch in nichthepatischen Fibrosen gebildet werden. Deswegen werden häufig direkte und indirekte Marker kombiniert. Tabelle 2 fasst die am besten validieren Markerkombinationen zusammen.

Die von der Ätiologie unabhängige diagnostische Qualität einfacher klinischer oder labormedizinischer Befunde wurde in einer großen Meta-Analyse von 86 Studien untersucht, welche von den Autoren als gut qualifiziert
Tabelle 2 Kombinationen von Serumbiomarkern für die Diagnostik der Leberfibrose [5, 6].

\begin{tabular}{|c|c|}
\hline Score & Komponenten \\
\hline APRI & AST/Plättchen Ratio \\
\hline Forn Index & Alter, Plättchen, $\gamma \mathrm{GT}$, Cholesterin \\
\hline Bonacini & Plättchen, AST, ALT, INR \\
\hline GUCl & AST, INR, Plättchen \\
\hline HALT C & Hyaluronsäure, TIMP1, Plättchen \\
\hline LOK-Index & AST, ALT, INR, Plättchen \\
\hline MP3 & TIMP1, P3NP \\
\hline ViraHep & Rasse, Alter, AST, Plättchen, alkal. Phosphatase \\
\hline FIB-4 & AST, ALT, Plättchen \\
\hline ELF plus $^{\circledR}$ & $\begin{array}{l}\text { Hyaluronsre, TIMP1, P3NP, BMI, T2DM / IFG, AST, } \\
\text { ALT, Plättchen, Albumin }\end{array}$ \\
\hline Fibrometer ${ }^{\circledR}$ & Alter, Gewicht, FPG, AST, ALT, Ferritin, Plättchen \\
\hline FibroSpect ${ }^{\circledast}$ & $\alpha 2$-Makroglobulin, Hyaluronat, TIMP1 \\
\hline FibroTest $^{\circledR}$ & $\begin{array}{l}\alpha 2 \text {-Makroglobulin, Apolipoprotein A-I, } \\
\text { Haptoglobin, Bilirubin, } \gamma \mathrm{GT}, \text { ALT }\end{array}$ \\
\hline HepaScore ${ }^{\circledR}$ & $\begin{array}{l}\text { Alter, Geschlecht, } \alpha 2 \text {-Makroglobulin, Hyaluronat, } \\
\text { TIMP1, } \gamma \mathrm{GT}\end{array}$ \\
\hline
\end{tabular}

wurden und in denen Leberzirrhose durch Biopsien verifiziert wurde [6]. Insgesamt wurden 19.533 Patienten in die Meta-Analyse eingeschlossen, von denen 4725 eine durch Biopsie nachgewiesene Zirrhose hatten (Prävalenz: 24\%; 95\% CI, 20\%-28\%). Etliche Befunde körperlicher Untersuchungen oder einfacher Laboruntersuchungen erhöhen die Wahrscheinlichkeit einer Zirrhose, nämlich das Vorhandensein eines Aszites (Likelihood-Ration LR=7,2, 95\% CI: 2,9-12), einer Plättchenzahl $<160^{\star} 10^{3} / \mu \mathrm{L}(\mathrm{LR}=6,3,95 \%$ CI: 4,3-8,3), Spinnen- Nävi (LR, 4,3, 95\% CI: 2,4-6,2) oder die Kombination einfacher Labortest-Ergebnisse, z. B. ein Bonacini Cirrhosis Discriminant Score $>7$ (LR, 9,4; 95\% CI: 2,6-37) oder ein APRI Index >2 (LR4,6, 95\% CI: 3,2-6,0). Für den Ausschluss einer Zirrhose stellten sich ein Lok index $<0,2$ (LR=0,09; 95\% CI: 0,03-0,31), eine Plättchenzahl $>160^{\star} 10^{3} / \mu \mathrm{L}$ (LR=0,29; 95\% CI: 0,20-0,39) und das Fehlen einer Hepatomegalie (LR=0,37, 95\% CI: 0,24-0,51) als die am besten geeigneten Merkmale heraus. Insgesamt war der klinische Gesamteindruck eines Arztes weniger informativ als die einzelnen oder kombinierten labormedizinischen Befunde, insbesondere beim Ausschluss einer Zirrhose (Positive LR 4,8, 95\% CI, 2,5-7,2; Negative LR 0,52, 95\% CI, 0,33-0,71) [6].

Die Biomarker und die aus ihnen abgeleiteten Algorithmen wurden insbesondere bei Patienten mit Hepatitis B oder C sowie bei Patienten mit nichtalkoholischer Fettleberkrankheit (NAFLD) evaluiert. Das Monitoring der Leberkrankheit von Patienten mit Hepatitis B oder C ist wichtig, um die Prognose zu bestimmen und antivirale Therapien $\mathrm{zu}$ indizieren. Insgesamt sind die Biomarker 
verlässlicher beim Nachweis einer Zirrhose als beim Nachweis intermediärer Fibrosestadien [1-5]. Am häufigsten validiert wurden APRI (ein freier, nichtpatentierter Algorithmus, in den die Messgrößen AST und Plättchenzahl eingehen) und der FibroTest ${ }^{\circledR}$ (ein patentierter Test, der in Frankreich verbreitet ist und in den die Messgrößen $\gamma \mathrm{GT}$, Haptoglobin, Bilirubin, Apolipoprotein A-I und $\alpha 2-$ Makroglobulin eingehen).

Eine Meta-Analyse der Ergebnisse des APRI-Tests bei 6259 HCV Patienten aus 33 Studien fand mittlere AUROC Werte von 0,77 und 0,83 für den Nachweis einer signifikanten Fibrose bzw. Zirrhose [7]. Eine Meta-Analyse des FibroTestsR bei 3501 Patienten mit Hepatitis C Virus [HCV] - und 1457 Patienten mit Hepatitis B-Virus [HBV]) Infektion [8] fand eine standardisierte AUROC von 0,84 für die Diagnose einer signifikanten Fibrose, ohne signifikanten Unterschied zwischen HCV (AUROC $=0,85$ ) und HBV (AUROC=0,80). Der Anteil diskordanter Ergebnisse von Biopsie und FibroTest ${ }^{\circledR}$ beträgt ca. $25 \%$. Wie zuvor dargelegt, ist die diagnostische Qualität der Biomarker auch durch prä-analytische und analytische Fehler der Biopsie begrenzt. Die Autoren des FibroTest ${ }^{\circledR}$ schätzen, dass die diskordanten Ergebnisse je zur Hälfte durch Fehler der Biopsie und des Biomarker-Tests verursacht werden. Sie entwickelten deswegen das Konzept des „Risikos falschpositiver und falsch-negativer Ergebnisse“ (RFPFN) und evaluierten die Ergebnisse ihrer Datenbank von nahezu 370.000 Messergebnissen [9]. In der Allgemeinbevölkerung betrug RFPN $0,5 \%-1 \%$. Es stieg auf $2 \%$ und mehr in Hochrisikogruppen wie Patienten von Tertiären Versorgungszentren oder HIV-Zentren oder bei Afrikanern mit sub-saharan Ursprung. Unter den Laborparametern trugen eine tiefe Konzentration von Haptoglobin $(0,46 \%)$ und eine hohe Konzentration von ApoA-I ( $0,2 \%)$ am stärksten zu RFPFN bei. Insgesamt schlossen die Autoren aus ihren Daten auf eine Anwendbarkeit des FibroTestR bei $99 \%$ der Patienten insgesamt und $97 \%$ der Hochrisiko-Patienten.

In einer direkten Vergleichsstudie bei 913 HCV- und 284 HBV-Patienten unterschieden sich die diagnostischen Qualitäten der drei patentierten Tests (FibroTest ${ }^{\circledR}$, Fibrometer $^{\circledR}$ und Hepascore ${ }^{\circledR}$ ) und des nicht-patientierten APRI-Tests nicht. Die AUROC-Werte rangierten zwischen 0,72 und 0,78 bei signifikanter Fibrose, sowie zwischen 0,77 und 0,86 bei Zirrhose [10]. In einer anderen Vergleichsstudie von neun Biomarker-Tests bei 436 Patienten mit Hepatitis $C$ betrugen die AUROC-Werte beim Nachweis einer signifikanten Fibrose $(\mathrm{F}>2)$ für nichtpatentierte Tests (Hyaluronsäure, Forn's Score, APRI, MP3, FIB-4, ELFG) 0,75 bis 0,78 und für patentierte Tests (FibroTest ${ }^{\circledR}$, Fibrometer $^{\circledR}$, Hepascore ${ }^{\circledR}$ ) 0,80 bis 0,82. Für den Nachweis einer Zirrhose betrugen die entsprechenden AUROCRanges 0,83 bis 0,88 bzw. 0,86 bis 0,89 [11].

Fast alle Modelle zur Beurteilung der Wertigkeit von klinischen oder histologischen Methoden zur Beurteilung des Schweregrades einer Fibrose fußen auf Baseline-Befunden aus Klinik, Labor oder Pathologie. Da sich der Schweregrad und auch die klinischen und labormedizinischen Surrogate einer Lebererkrankung über die Zeit ändern, sollten dynamische Modelle eine bessere prognostische Wertigkeit haben. Mit dieser Hypothese wurde aus den Daten der Hepatitis C Long-Term Treatment Against Cirrhosis (HALT-C) Studie zwei Modelle entwickelt, um die klinische Dekompensation (Modell 1) bzw. die Lebertransplantation oder den hepatogenen Tod (Modell 2) vorauszusagen. Bei 470 Patienten sagte ein Algorithmus aus Plättchenzahl, AST/ALT-Ratio, Bilirubin bei Baseline sowie starken Veränderungen der Plättchenzahl und der Bilirubin- und Albuminspiegel die klinische Dekompensation bei 60 Patienten am besten voraus (Modell 1). Bei 483 Patienten waren ein Algorithmus aus Plättchenzahl und Albumin-Konzentration bei Baseline sowie die Verschlechterung der AST/ALT-Ratio und der Abfall der Albuminkonzentration die besten Prädiktoren von 79 Transplantationen oder leberbedingten Todesfällen (Modell 2) [12].

\section{Bildgebende Verfahren}

Die Aktivierung von hepatischen Sternzellen und die Bildung extrazellulärer Matrix führen zu Änderungen der Mikrostruktur in der Leber, welche sich durch abnehmende Elastizität und verändertem Blutfluss zeigen [1-4]. Die Veränderungen in der Elastizität können durch sonografische Elastografie (FibroScan ${ }^{\mathrm{TM}}$ ), Magnetresonanztomografie (MRI) oder Akustischen Strahlenkraft Impuls (ARFI=acoustic radiation force impulse) nicht-invasiv gemessen werden.

Bei der Transienten Ultraschall Elastographie (FibroScan $^{\mathrm{TM}}$ ) wird die Elastizität des Leberparenchyms durch einen $5 \mathrm{MHz}$ Ultraschall und die Auslösung niedrigfrequenter elastischer Wellen mittels eines speziellen Ultraschall-Vibrators gemessen. Dabei wird die Ausbreitungsgeschwindigkeit von Puls-Echo-Wellen mittels Ultraschall-Bildgebung gemessen. Weil fibrotisches Gewebe härter als gesundes Leberparenchym ist, korreliert die Aufbreitungsgeschwindigkeit mit dem Ausmaß der Fibrose. FibroScan ${ }^{\mathrm{TM}}$ ist in Europa für die klinische Diagnostik zugelassen. Die Methode wurde bei verschiedenen Lebererkrankungen evaluiert, wie Hepatitis B und 
C, Alkoholische Lebererkrankung und NAFLD. MetaAnalysen zeigten die Zuverlässigkeit von FibroScan ${ }^{\mathrm{TM}}$ für die Diagnose einer signifikanten Fibrose (F2, AUC $=0,84)$, schweren Fibrose $(\mathrm{F} 3, \mathrm{AUC}=0,89)$ und Zirrhose (F4, AUC=0,94). Jedoch variierte bei F2 die AUC stark in Abhängigkeit der zugrundeliegenden Erkrankung. Bei der Diagnose der Zirrhose ist die FibroScan-Technologie durch die Aktivität von Nekrose und Entzündung bei NAFLD-Patienten eingeschränkt. Weitere die Qualität einschränkende Faktoren sind die Expertise des Untersuchers, das Alter des Patienten, das Vorhandensein von Ascites, ein Body Mass Index $\geq 28 \mathrm{~kg} / \mathrm{m}^{2}$ und viszerale Adipositas, sowie die Weite der Interkostalräume. Als Ergebnis konnte in einer großen Studie in 20\% der Untersuchungsfälle kein Ergebnis erzeugt werden [2].

ARFI kombiniert konventionellen Ultraschall mit einer lokalen Untersuchung der Leberelastizität. Insgesamt korrelieren die Ergebnisse von FibroScan ${ }^{\mathrm{TM}}$ und ARFI gut. Im Vergleich zu FibroScan ${ }^{\mathrm{TM}}$ ist bei ARFI der limitierende Einfluss von anatomischen Hindernissen (z. B. große Gefäße) oder Steatose minimiert. Ein weiterer Vorteil ist die mögliche Integration der ARFI Software in herkömmliche Ultraschallgeräte, wodurch die bei FibroScan ${ }^{\mathrm{TM}}$ teure Investition entfällt [2].

Durch MRI können sowohl die Leber-Elastizität als auch Veränderungen der Wasser-Diffusion, welche bei Zirrhose vorkommen, gemessen werden. Bei der Magnetischen Resonanz Elastographie (MRE) werden die sich durch das Lebergewebe ausbreitenden akustischen Scherwellen durch drei Schritte direkt visualisiert und quantifiziert: 1) Generation mechanischer Wellen im Gewebe; 2) Bildgebung der micron level Verlagerungen, welche durch die sich ausbreitenden Wellen verursacht werden mittels einer speziellen MRI Technik (oscillating motion-sensitizing Gradients); und 3) Prozessierung der Wellenbilder mittels eines Algorithmus, der quantitative Landkarten der physikalischen Lebereigenschaften herstellt. Dadurch stellt MRE die Verteilung des Bindegewebes in der Leber dar. Im Vergleich zur Biopsie zeigt MRE eine sehr gute Übereinstimmung beim Nachweis des Fibrosegrades: Im Vergleich zu FibroScan ${ }^{\mathrm{TM}}$ zeichnet sich MRE dadurch aus, dass es auch den niedrigsten Fibrosegrad erfasst und dass die Ausfallquote kleiner ist: 94\% statt $84 \%$ der Untersuchungen können durchgeführt werden. Nur sehr stark übergewichtige Patienten, die nicht in die MR-Maschine passen, sind ein Hinderungsgrund [2].

In der ANRS HCEP23-Studie wurde bei nahezu 500 Patienten mit chronischer Hepatitis $\mathrm{C}$ die diagnostische Wertigkeit von transienter Elastographie (FibroScan ${ }^{\mathrm{TM}}$ ) und neun Blut-Biomarkern verglichen: Fibrometer ${ }^{\circledR}$, FibroTest $^{\circledR}$, Forn’s Score, APRI, MP3, ELFG, Hepascore ${ }^{\circledR}$,
Fib4 und Hyaluronsäure. Die Elastographie war bei $22 \%$ der Patienten nicht auswertbar, sodass für 382 Patienten alle Verfahren verglichen werden konnten [11]. Für die Diagnose einer signifikanten Fibrose zeigte Fibroscan ${ }^{\circledR}$ mit einer AUROC von 0,82 keine Überlegenheit gegenüber den patentierten Biomarkern (0,81 bei FibroTest ${ }^{\circledR}$, 0,82 bei Hepascore ${ }^{\circledR}$ und 0,83 bei Fibrometer ${ }^{\circledR}$ ) wohl aber gegenüber den klinischen Scores $(0,74-0,78)$. Die Elastographie war den Biomarkern beim Nachweis einer Zirrhose überlegen [AUROC=0,93 gegenüber 0,84 (Fib4) bis $0,90\left(\right.$ Fibrometer $\left.^{\circledR}\right)$ ] bei den Biomarkern. Die Autoren berechneten auch die Zahl der durch Bildgebung oder Biomarker vermeidbaren Biopsien unter der Vorgabe von $90 \%$ cut-offs für negative und positive prädiktive Werte. Die Unterschiede zwischen Fibrometer ${ }^{\circledR}(36,6 \%)$, Fibrotest $^{\circledR}(35,6 \%)$, Hepascore ${ }^{\circledR}(30,5 \%)$ und FibroScan $(45,8 \%)$ waren nicht statistisch signifikant [11].

Die nicht optimale Diagnostik der signifikanten Fibrose sowohl durch Biomarker als auch durch Bildgebung legt nahe, beide Strategien zu kombinieren. Tatsächlich wurde dieses Konzept in ersten Studien verfolgt. In der zuvor beschriebenen Studie von Zarski et al. erhöhte die Kombination von Elastographie und patentierten Biomarkern den Anteil korrekt klassifizierter Patienten mit einer signifikanten Fibrose (F2) von 70\%-73\% auf $80 \%-83 \%$ [11]. In einer anderen Studie mit Patienten mit chronischer Hepatitis C wurden 97\% der Patienten durch einen Kombinations-Algorithmus aus FibroTest ${ }^{\circledR}$ und APRI richtig klassifiziert und hätten theoretisch 48\% der Biopsien vermieden werden können. Die Kombination von Elastographie und Fibrotest hätte sogar 79\% der Biopsien vermieden [13]. Ähnliche Ergebnisse fanden sich bei Kombination von Elastographie und Fibrometer ${ }^{\circledR}$ [14].

Diese vielversprechenden Ergebnisse zur nicht-invasiven Diagnostik der Fibrose spiegeln sich in den neuesten Empfehlungen der European Association for the Study of Liver Diseases on Chronic Hepatitis $C$ wider, welche den Einsatz von nicht-invasiven Methoden zu Diagnostik und Monitoring der Fibrose bei chronischer Hepatitis C empfehlen [15]. Abbildung 1 stellt einen Klinischen Pfad zur Umsetzung dieses Konzeptes vor.

\section{Definitionen und Epidemiologie der Nicht-alkoholischen Fettleber- Krankheit (NAFLD)}

Die Diagnose NAFLD erfordert erstens den Nachweis einer Steatose durch Histologie oder Bildgebung und 


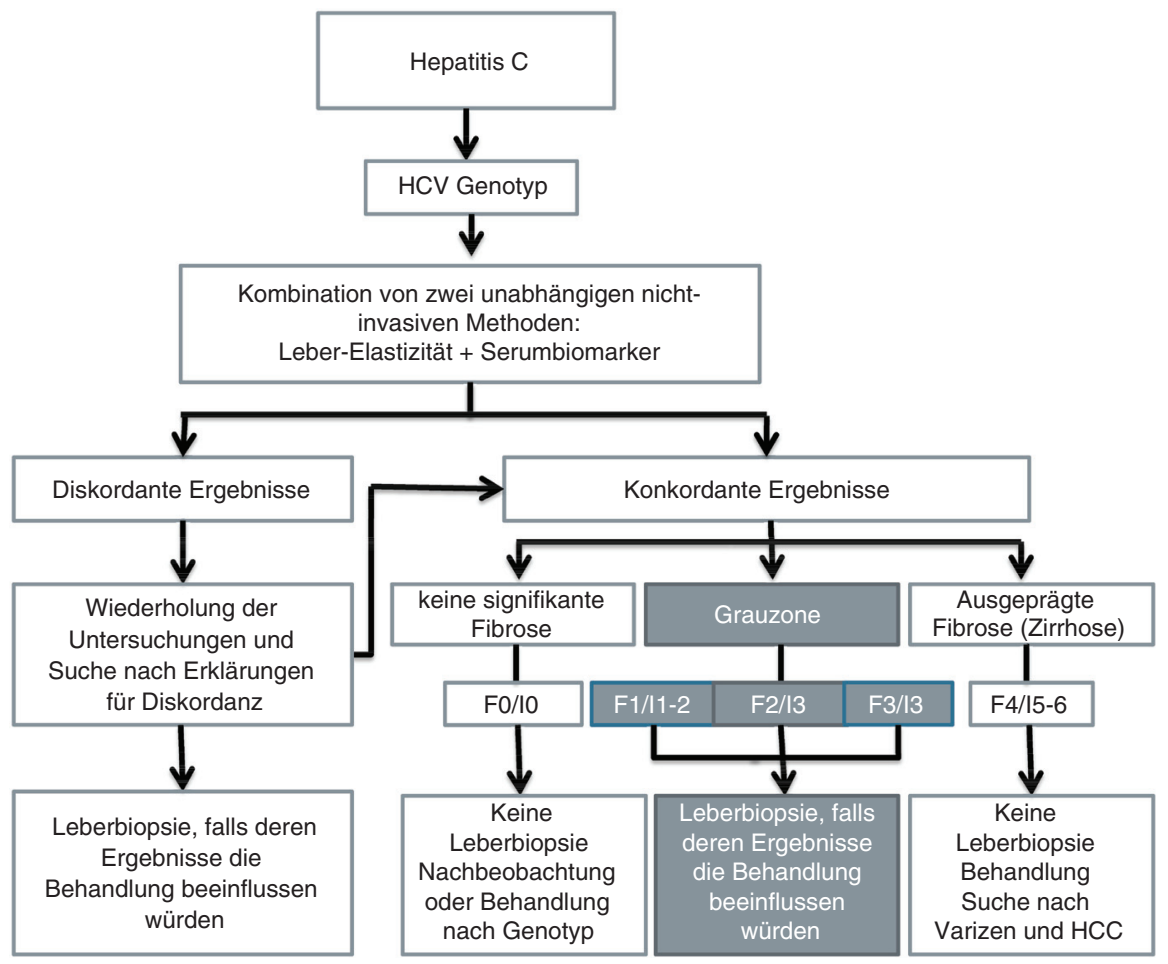

Abbildung 1 Vorschlag eines Klinischen Pfades für den Einsatz nicht-invasiver Methoden zur Risiko- und Therapiestratifizierung der chronischen Hepatitis $C$.

F0-F4=Metavir-Stadien der Fibrose; 10-16=Ishak Stadien der Fibrose. Übersetzt auf Referenz [3].

zweitens den Ausschluss von Ursachen für eine sekundäre Leberverfettung wie übermäßigen Alkoholkonsum, die Einnahme steatogener Medikamente oder hereditärer Speicherkrankheiten (Tabelle 3). Histologisch wird NAFLD in NAFL (Nicht-alkoholische Steatose oder Fettleber) und

Tabelle 3 Ursachen einer sekundären Steatose [16].

Makrovesikuläre Steatose

- Exzessiver Alkoholkonsum

- Hepatitis C (Genotyp 3)

- Wilson Krankheit

- Lipodystrophie

- Hunger

- Parenterale Ernährung

- Abetalipoproteinämie

- Medikamente (z. B. Amiodaron, Methotrexat, Tamoxifen, Korticosteroide)

Microvesikuläre Steatose

- Reye Syndrom

- Medikamente (Valproat, anti-retroviral Medikamente)

- Akute Schwangerschaftsfettleber

- HELLP Syndrom

- Angeborene Stoffwechselerkrankungen (z. B. LCAT Defizienz, Cholesterylester Speicherkrankheit/ Wolman Krankheit)
NASH (nicht-alkoholische Steatohepatitis oder Fettleberhepatitis) differenziert (Tabelle 4) [16].

Häufig haben Patienten mit NAFLD Übergewicht oder Adipositas, Diabetes mellitus sowie eine durch Hypertriglyzeridämie und niedriges HDL-Cholesterin gekennzeichnete Dyslipidämie. Entsprechend wird NAFLD heute als die hepatische Manifestation des Metabolischen Syndroms verstanden. NAFLD kommt häufig auch mit anderen mit Metabolischem Syndrom assoziierten Krankheiten und Symptomen vor, z. B. Polycystischem Ovarsyndrom,

Tabelle 4 Stadien-Einteilung einer nicht-alkoholischen Fettleberkrankheit (NAFLD) [16].

- Nichtalkoholische Fettleber-Krankheit (NAFLD): Überbegriff für alle Manifestationen (Steatose=NAFL), Steatohepatitis (NASH), Fibrose, Zirrhose

- Nichtalkoholische Fettleber (Steatose=NAFL): Leberverfettung ohne Hinweise auf Leberzellnekrose (Ballonierung) oder Fibrose (Brückenbildung): geringe Risiken für Zirrhose oder Leberversagen

- Nichtalkoholische Fettleber-Hepatitis (Steatohepatitis=NASH): Leberverfettung mit Entzündungszeichen: Leberzellnekrosen (Ballonierung) und/oder Fibrose (Brückenbildung): Risiken für Zirrhose oder Leberversagen. Leberzell-Karzinom eher selten 
Schlafapnoe-Syndrom und männlichem Hypogonadismus [16]. Klinische Relevanz hat die Diagnose durch die durch NAFLD erhöhten Risiken für die Manifestation eines Diabetes mellitus, einer koronaren Herzkrankheit, einer chronischen Nierenkrankheit sowie durch die Progression der NAFLD bis zur Zirrhose und schließlich zum hepatozellulären Karzinom [16-19].

Die Diagnose NAFLD wird mit der zunehmenden Prävalenz von Adipositas und Metabolischem Syndrom immer häufiger gestellt. In vielen Ländern gilt NAFLD als die häufigste Ursache erhöhter Leberenzym-Aktivitäten im Plasma. Allerdings variieren die epidemiologisch dokumentierten Prävalenzen der NAFLD in Abhängigkeit der eingesetzten Diagnosemethoden und -kriterien erheblich zwischen $3 \%$ und 50\%. In zwei Histologie-Studien von Lebend-Leberspendern betrug die Prävalenz 20\% und $51 \%$ [20, 21]. In einer texanischen Bevölkerungsstudie von mittelalten Personen, die Leberfett mittels Magnetresonanzspektroskopie maß, aber die Alkoholanamnese nicht berücksichtigte, fand sich eine Prävalenz von 31\% [22]. In einer anderen amerikanischen Studie [23], welche die Steatose sonografisch erfasste und die Alkoholanamnese berücksichtigte, betrug die Prävalenz von NAFLD $46 \%$, wovon nahezu 30\% NASH hatten (12,2\% Gesamtprävalenz). Im National Health and Nutrition Examination Survey III (NHANES III), der zwischen 1988 und 1994 durchgeführt wurde, betrug die Prävalenz von NAFLD bei 11.613 Teilnehmern fast 19\% (sonografische Steatose bei Ausschluss von chronischem Alkoholkonsum und Hepatitis B und C). Fast 12\% der NAFLD-Personen bzw. mehr als 2\% der Gesamtpopulation hatten NASH [24]. Innerhalb der Gesamtbevölkerung ist NAFLD mit Geschlecht, Alter und Ethnizität assoziiert: Männer sind häufiger betroffen als Frauen; die Wahrscheinlichkeit fortgeschrittener Stadien (NASH, Zirrhose) steigt mit dem Alter, Übergewicht/Adipositas und dem Vorhandensein einer Hypertonie [24]; Hispanische Weiße sind häufiger betroffen als nicht-hispanische Weiße, welche wiederum häufiger betroffen sind als Afrikaner oder Ur-Amerikaner [16, 24].

Die Prävalenzen der NAFLD sind in Risikogruppen noch höher als in der Normalbevölkerung. Im NHANES III betrug die Prävalenz von NAFLD bei 7156 übergewichtigen oder adipösen Personen 27,8\% gegenüber 7,4\% bei 4457 schlanken Teilnehmern. Interessanterweise unterschieden sich die unabhängigen Prädiktoren für NAFLD bei Schlanken und Übergewichtigen: Sie sind jünger und eher weiblich und haben weniger Komponenten des Metabolischen Syndroms [24]. Mehr als 90\% der massiv adipösen Patienten, welche sich einer bariatrischen Chirurgie unterziehen, haben eine NAFLD. Bei bis 5\% findet sich sogar eine Zirrhose, die häufig präoperativ unbekannt ist [16]. Bei Patienten mit Diabetes mellitus Typ 2 (T2DM) schwanken die Angaben zur Prävalenz zwischen 69\% und $87 \%$ [16]. Bereits in prädiabetischen Patienten mit Impaired Fasting Plasmaglucose oder Glukose-Intoleranz ist die Prävalenz von NAFLD erhöht [17, 18]. Bei Patienten einer Lipidklinik beträgt die NAFLD-Prävalenz 50\%.

\section{Praxis-Empfehlungen zu Diagnostik und Management der NAFLD}

Die American Gastroenterological Association, die American Association for the Study of Liver Diseases und das American College of Gastroenterology haben in 2012 gemeinsame Praxis-Empfehlungen zu Diagnostik und Management der NAFLD publiziert [16]. Ein weiteres Postionspapier der European Society for Pediatric Gastroenterology, Hepatology, and Nutrition (ESPGHN) beschäftigte sich mit der NAFLD im Kindes- und Jugendalter [25].

Wie heute üblich und durch das AASLD Practice Guidelines Committee empfohlen, werden die insgesamt 45 Praxisempfehlungen der amerikanischen Gastroenterologen und Hepatologen nach GRADE (Grading of Recommendation Assessment, Development, and Evaluation) als stark (1) oder schwach (2) klassifiziert. Zudem werden die Empfehlungen nach der Qualität ihrer Evidenz gewichtet: hoch (A), moderat (B) und gering (C). Nachfolgend sind nur die für die Diagnostik relevanten Empfehlungen zusammengefasst [16]. Die Ziffern und Buchstaben in Klammern verweisen auf GRADE-Klassifizierung bzw. Qualität der Evidenz, wie zuvor beschrieben.

- Bei der Abklärung eines Verdachts auf NAFLD ist es essentiell, andere Ursachen einer Fettleber und koexistierende Lebererkrankungen auszuschließen (1A).

- Patienten mit unerwartetem Steatose-Befund durch Bildgebung, die auch klinische oder labormedizinische Hinweise auf eine Hepatopathie haben, sollen differentialdiagnostisch abgeklärt werden (1A). Zur Abgrenzung von alkoholischer und nichtalkoholischer Lebererkrankung wird ein Konsum von durchschnittlich >21 Drinks/Woche bei Männern und $>14$ Drinks/Woche bei Frauen definiert (2C). Bei persistierend hohen Ferritin-Spiegeln und Transferrinsättigung soll eine Hämochromatose genetisch (HFE 282Y-Homzygotie) oder bioptisch ausgeschlossen werden (1B). Bei Vorhandensein von Auto-Antikörpern oder anderen Hinweisen auf ein Autoimmunhepatitis (sehr hohe ALT Aktivität, starke $\gamma$-Globulin-Vermehrung) sollen alle Untersuchungen 
zum Ausschluss einer Autoimmunhepatitis konsequent durchgeführt werden (1B).

- Patienten mit unerwartetem Steatose-Befund durch Bildgebung, die keine klinischen oder labormedizinischen Hinweise auf eine Hepatopathie haben, sollen in Bezug auf metabolische Risikofaktoren (Übergewicht, Adipositas, Diabetes und Glukoseintoleranz, Dyslipidämie) und Ursachen einer sekundären Fettleber (Alkohol, Medikamente) abgeklärt werden (1A). Bei ihnen soll keine Leberbiopsie durchgeführt werden (1B).

- Bei erwachsenen Patienten der Primärversorgung oder von Diabetes- und Adipositas-Kliniken ist ein Screening auf NAFLD derzeit nicht empfohlen, weil Langzeitnutzen und Kosteneffektivität unbekannt sind (1B). Aus denselben Gründen ist ein systematisches Screening von Familienangehörigen auf NAFLD nicht empfohlen (1B).

- Der NAFLD Fibrosis Score (www.nafldscore.com) ist hilfreich, um NAFLD Patienten mit schwerer Fibrose (Brückenbildung) oder Zirrhose zu identifizieren (1B). Die Plasmakonzentration von Cytokeratin 18-Fragmenten (CK-18) ist ein vielversprechender Biomarker für die Identifizierung von Patienten mit NASH oder fortgeschrittener Fibrose. Trotzdem ist es zu früh, um den Marker für die klinische Routine zu empfehlen (1B).

- Das Vorhandensein eines Metabolischen Syndroms macht bei NAFLD Patienten eine Steatohepatitis wahrscheinlich. Entsprechend soll eine Biopsie erwogen werden (1B). Das Vorhandensein von Metabolischem Syndrom und hohem NAFLD Fibrose Score können für die Erkennung von Patienten mit hohem Risiko von NASH oder fortgeschrittener Fibrose verwandt werden (1B). Leberbiopsien sollen erwogen werden bei NAFLDPatienten mit erhöhtem Risiko für NASH oder fortgeschrittener Fibrose (1B). Leberbiopsien sollen auch erwogen werden für Patienten mit Verdacht auf NAFLD, bei denen konkurrierende Ursachen ohne Leberbiopsie nicht ausgeschlossen werden können (1B).

- Patienten mit NASH-Zirrhose sollen auf das Vorhandensein von Ösophagus-Varizen gescreent werden (1B). Patienten mit NASH-Zirrhose sollen auf die Entwicklung eines hepatozellulären Karzinoms überwacht werden (1B). Routinemäßige Wiederholungen von Leberbiopsien sind bei Patienten mit NAFL oder NASH nicht empfohlen (2C).

- Bei nicht-übergewichtigen Kindern mit Fettleber sollen neben den auch für Erwachsene empfohlenen Ursachen-Abklärungen auch monogene Ursachen gesucht werden, nämlich Störungen der Fettsäure-Oxidation, lysosomale Speicherkrankheiten und peroxisomale Störungen (2C). Niedrige Auto-Antikörpertiter kommen bei Kindern mit NAFLD häufig vor. Hohe Titer, vor allem in Kombination mit ausgeprägten Erhöhungen der ALT und $\gamma$-Globuline, sollten Anlass zu einer Leberbiopsie geben, um eine Autoimmunhepatitis auszuschließen (2B). Ein systematisches Screening auf NAFLD bei übergewichtigen oder adipösen Kindern wird mangels Evidenz eines Nutzens nicht empfohlen. Dies ist im Gegensatz zu einem anderen Expertengremium, das eine zweijährliche ScreeningUnterschung von Leberenzymen empfahl (1B). Bei Kindern wird eine Leberbiopsie angeraten, wenn die Diagnose unsicher ist, wenn multiple Diagnosen möglich erscheinen oder bevor eine potenziell hepatotoxische Medikation begonnen wird (1B). Bei Kindern ist eine Biopsie auch angeraten, bevor eine Pharmakotherapie von NASH begonnen wird (2C). Pathologen sollen mit den histologischen Besonderheiten der NAFLD bei Kindern vertraut sein (1B).

\section{Einzelne Biomarker der NAFLD und NASH}

Der Goldstandard für die Diagnosen NAFLD und NASH ist die Leberbiopsie im Verbund mit dem Ausschluss von chronischem Alkoholkonsum und anderen Grundkrankheiten (Tabelle 3). Indirekte Hinweise ergeben sich durch klinische Zeichen und Symptome sowie Befunden von Labor- und Bildgebungsuntersuchungen, sowie die Kombination davon. Die meisten klinischen, labormedizinischen und radiologischen Untersuchungen sind nicht sensitiv und spezifisch genug, um NAFLD und NASH zu differenzieren und das Vorhandensein und das Ausmaß von Fibrose festzustellen [26-28].

Die meisten Patienten mit NAFLD sind asymptomatisch. Wenn überhaupt vorhanden, sind die Symptome und klinischen Untersuchungsbefunde unspezifisch. Mit größter Regelhaftigkeit werden Komponenten des Metabolischen Syndroms gefunden: Übergewicht oder Adipositas, Diabetes und/oder Bluthochdruck.

Es gibt keinen einzelnen Laborparameter, der die Diagnose NAFLD erlaubt oder zwischen Steatose, NASH und Fibrose differenziert. Obwohl erhöhte Aminotransferasen-Aktivitäten häufig den Verdacht NAFLD wecken, so sind bei bis zu 78\% der NAFLD Patienten die Leberenzym-Aktivitäten normal. ALT- und AST-Aktivitäten sind maximal auf das Vierfache des oberen Referenzbereiches erhöht. Bei Patienten ohne oder mit geringer Fibrose ist die ALT/AST-Ratio normalerweise $<1$, bei Zirrhose $>1$. Die Aktivität der gamma-Glutamyltransferase $(\gamma \mathrm{GT})$ ist häufig 
bei Patienten mit NAFLD erhöht. Eine erhöhte $\gamma \mathrm{GT}$ ist bei NAFLD-Patienten mit Fibrose und erhöhtem Mortalitätsrisiko assoziiert. Bei einem cut-off von $100 \mathrm{U} / \mathrm{L}$ betragen Sensitivität und Spezifität ca. 80\% bzw. 70\% [26-28].

In den letzten Jahren wurde eine erhöhte ApoptoseRate als typisches Zeichen der NASH gefunden. Dieses Wissen führte zur Entdeckung von Fragmenten, welche durch enzymatische Spaltung des Cytokeratins-18 (CK-18) durch die Caspasen 3 und 7 entstehen und ins Plasma freigesetzt werden [29]. Plasmaspiegel von CK-18-Fragmenten sind bei Patienten mit NASH erhöht und ermöglichen deren Differenzierung von Steatose. CK-18 Fragmente ist anderen Kandidaten-Biomarkern, z. B. dem Adipozyten Fatty Acid Binding Protein (FABP4) oder dem Fibroblast Growth Factor 21 (FGF21), sowohl bei der Differenzierung von NAFLD Patienten von Kontrollen (AUROC $=0,91$ im Vergleich zu 0,66 bzw. 0,84) als auch bei der Differenzierung von Steatosie und NASH überlegen (AUROC $=0,70$ im Vergleich zu 0,59 bzw. 0,62) [30]. In mehreren Studien wiesen CK-18-Fragmente eine AUROC von 0,80 bis 0,93 auf, wobei an den optimalen diagnostischen cut-offs die Spezifität $81 \%-100 \%$ und die Sensitivität $62 \%-82 \%$ betrugen. In der bislang größten und einzigen multizentrischen Validierungsstudie betrug die AUROC 0,83 (ALT zum Vergleich: 0,58 ) bei einer Sensitivität von $75 \%$ und einer Spezifität von $81 \%$ am idealen cut-off [31]. Allerdings unterscheiden sich die auf dem Markt befindlichen CK-18 Fragment Immunoassays bezüglich ihrer Spezifität für Caspasegespaltenes CK-18, was möglicherweise zur beträchtlichen Variation der diagnostischen Effizienz in den bisherigen Studien führte. Eine Hannoveraner Arbeitsgruppe verglich deswegen bei 121 NAFLD-Patienten einen sogenannten M30-ELISA, der ausschließlich Caspase-gespaltene CK-18-Fragmente erkennt und damit Leberzell-Apoptose misst, mit dem M65-ELISA, der sowohl gespaltenes als auch ungespaltenes CK-18 erkennt und damit Zelltod insgesamt misst. Sowohl bei der Differenzierung von Steatose und NASH als auch bei der Differenzierung leichter Fibrosegrade zeigte der M65-ELISA eine bessere Performance als der M30-ELISA [32].

\section{Biomarker-Panel und Algorithmen für die Diagnostik und Stratifizie- rung der NAFLD}

In den letzten Jahren wurden mehrere Algorithmen und Scores entwickelt, um mit einfachen Laborparametern und klinischen Informationen NAFLD und NASH zu diagnostizieren und letztere in Fibrose-Stadien einzuteilen.
Der NAFLD Liver Fat Score, der Fatty Liver Index (FLI) und der Hepatic Steatosis-Index (HSI) wurden entwickelt, um die Patienten mit Steatose zu identifizieren. Der NAFLD-Liver Fat Score enthält als Variablen das Vorhandensein des Metabolischen Syndroms oder Diabetes mellitus Typ 2, Nüchterninsulin, AST sowie die AST/ALT-Ratio und zeigte eine AUROC 0,86-0,87. Der Fatty Liver Index berücksichtigt Body Mass Index (BMI), Bauchumfang, Triglyzerid-Spiegel und erzeugt damit eine Punkteskala von 0-100. Die AUROC betrug 0,84. Der HSI integriert ALT, AST, BMI, Alter und Geschlecht und erreichte in einer Kohorte von über 5000 Personen eine AUROC von 0,81 [28].

Eine ganze Reihe von Scores und Algorithmen wurde entwickelt, um NASH zu diagnostizieren. Im Median betrugen deren AUROCs 0,76. Einige dieser Algorithmen nutzen einfache Informationen, z. B. der HAIR Score, der Hypertension, ALT und Insulin-Resistenz integriert, oder ein klinisches Modell, das Alter, Geschlecht, BMI, AST, AST/ALT-Ratio und Hyaluronsäure integriert. Kompliziertere Modelle, welche teilweise auch wenig verbreitete Laborparameter benötigen, z. B. der NashTest mit 13 Variablen (Alter, Geschlecht, Körpergröße und -gewicht sowie die Serumspiegel von Triglyzeriden, Cholesterin, $\alpha_{2}$-Makroglobulin, Apolipoprotein A-I, Haptoglobin, $\gamma \mathrm{GT}$, ALT, AST, und Gesamtbilirubin) oder ein von Younoussi et al. entwickeltes Modell (Diabetes, Geschlecht, BMI, Triglyzeride, CK-18-Fragmente und Gesamt CK-18) erreichten auch keine bessere diagnostische Leistung [26, 28].

Für die Erkennung und Stadieneinteilung der Fibrose bei NASH-Patienten wurden Algorithmen evaluiert, die entweder bei Patienten mit chronischer Hepatitis C (FibroTest $^{\circledR}$, Fibrometer ${ }^{\circledR}$, ELF, APRI) oder eigens für NAFLDPatienten (NAFLD-Fibrosis Score, BARD-Score) entwickelt wurden $[3,26,28]$. Tabelle 5 stellt die bei NAFLD ausgewerteten Scores zusammen. Die amerikanischen Gastroenterologen favorisieren in ihren Guidelines den NAFLD-Fibrose Score [16]. In einer Meta-Analyse zeigte der NAFLD-Fibrosis Score beim Nachweis einer NASH mit fortgeschrittener Fibrose $(\geq \mathrm{F} 3)$ betrugen AUROC sowie Sensitivität und Spezifität beim idealen cut-off 0,85, 0,90 und 0,97 [33]. Bei einem direkten Vergleich zeigte sich Fibrometer ${ }^{\circledR}$ beim Nachweis einer signifikanten Fibrose (>F2) mit einer AUROC von 0,94 gegenüber dem von den amerikanischen Gastroenterologen favorisierten NAFLD-Fibrose Score (AUROC 0,88) und APRI (AUROC $0,87)$ überlegen. Bei der Diagnose der Zirrhose bestand kein Unterschied (AUROCs: 0,90, 0,90, 0,84) [34]. In einer anderen Vergleichsstudie [35] wurden transiente Elastographie, AST/ALT-Ratio, APRI, FIB-4, BARD und NAFLDFibrose Score verglichen. Wie bei chronischer Hepatitis 
Tabelle 5 Biomarker-Algorithmen zur Diagnose der Fibrose bei NAFLD [3, 28, 33-35].

\begin{tabular}{|c|c|c|c|c|}
\hline Score & Komponenten & AUROC $\geq F 2$ & AUROC $\geq F 3$ & AUROC F4 \\
\hline AAR & AST/ALT-Ratio & & $0,66^{b}$ & $0,66^{\mathrm{b}}$ \\
\hline APRI & AST/Plättchen Ratio & $0,87^{\mathrm{a}}$ & & $0,84^{\mathrm{a}}$ \\
\hline FibroTest $^{\circledR}$ & $\begin{array}{l}\alpha 2 \text {-Makroglobulin, Apolipoprotein A-I, } \\
\text { Haptoglobin, Bilirubin, } \gamma \mathrm{GT} \text {, ALT }\end{array}$ & & 0,84 & \\
\hline Fibrometer $^{\circledR}$ & Alter, Gewicht, FPG, AST, ALT, Ferritin, Plättchen & $0,94^{\mathrm{a}}$ & & $0,90^{\mathrm{a}}$ \\
\hline FIB-4 & AST, ALT, Plättchen & & $0,80^{\mathrm{b}}$ & $0,81^{\mathrm{b}}$ \\
\hline ELF plus & $\begin{array}{l}\text { Hyaluronsäure, TIMP1, P3NP, BMI, T2DM / IFG, AAR, } \\
\text { Plättchen, Albumin }\end{array}$ & 0,84 & 0,93 & 0,98 \\
\hline NAFLD Score & Alter, Hyperglykämie, BMI, Plättchen, AAR, Albumin & $0,88^{\mathrm{a}}$ & $0,75^{b}$ & $\begin{array}{l}0,90^{\mathrm{a}} \\
0,80^{\mathrm{b}}\end{array}$ \\
\hline BARD & BMI, AAR, T2DM & & $0,69^{b}$ & $0,62^{b}$ \\
\hline
\end{tabular}

AAR, AST/ALT-Ratio; AUROC, Area under the Receiver Operator Characteristics Curve; BMI, Body Mass Index; FPG, Fasting plasma glucose; IFG, Impaired Fasting Glucose; P3NP, aminoterminales Peptid von Prokollagen III; T2DM, Diabetes mellitus Type 2; TIMP1, tissue inhibitor of metalloproteinase 1. Mit Asterisks gekennzeichnete AUROC-Werte kommen aus zwei vergleichenden Studien, in denen die jeweiligen Scores direkt gegeneinander verglichen wurden (a34; $\left.{ }^{\mathrm{B}} 35\right)$.

war die Bildgebung allen Biomarker Scores überlegen. Bei einer $\geq$ F3-Fibrose betrug die AUROC der transienten Elastographie 0,93 im Vergleich zu 0,66 (AST/ALT-Ratio) bis 0,80 (FIB4) bei den Biomarkern; bei einer Zirrhose betrugen die AUROCs 0,95 für Transiente Elastographie und 0,62 (BARD) bis 0,80 oder 0,81 (NAFLD-Score bzw. FIB-4) für die Biomarker.

Zusammengefasst gibt es leider keinen einzelnen Laborparameter, der die Diagnosen oder Stadien-Einteilungen von NAFLD oder NASH sicher ermöglicht. CK-18 ist der derzeit meistversprechende Einzelparameter für die Diagnose NASH. Entsprechend haben die Amerikanischen Gastroenterologen und Hepatologen CK-18 in ihren Empfehlungen hervorgehoben, allerdings ohne den Routineeinsatz zu proklamieren [16]. Weitere Validierungsstudien sind nötig. CK-18 ist derzeit nicht als Routine-Test verfügbar. Die Datenlage zur Wertigkeit der Scores und
Algorithmen zur Diagnostik und Schweregrad-Einteilung von NAFLD und NASH ist im Vergleich zur Wertigkeit der Scores für die chronische Hepatitis noch nicht so gut abgesichert. Dennoch scheinen die Fibrose-Scores gut geeignet, die Fibrose bei NAFLD abzuschätzen. Wiederum scheinen die patentierten Scores den freiverfügbaren inkl. des von den Guidelines favorisierten NAFLD-Fibrose Scores überlegen. Dem steht die einfachere Verfügbarkeit der letzteren gegenüber. Die mit konventionellen Markern erstellten Scores erscheinen auch dem CK-18 für die Diagnose von NASH unterlegen.

Interessenkonflikt: Der Autor erklärt, dass keine wirtschaftlichen oder persönlichen Interessenkonflikte bestehen.

Eingang 18.10.2013; Akzeptanz 21.2.2014; vorab online veröffentlicht 21.3.2014

\section{Literatur}

1. Martínez SM, Crespo G, Navasa M, Forns X. Noninvasive assessment of liver fibrosis. Hepatology 2011;53:325-35.

2. Baranova A, Lal P, Birerdinc A, Younossi ZM. Non-invasive markers for hepatic fibrosis. BMC Gastroenterol 2011;11:91.

3. Clark PJ, Patel K. Noninvasive tools to assess liver disease. Curr Opin Gastroenterol 2011;27:210-6.

4. Duarte-Rojo A, Altamirano JT, Feld JJ. Noninvasive markers of fibrosis: key concepts for improving accuracy in daily clinical practice. Ann Hepatol 2012;11:426-39.

5. Castera L. Noninvasive methods to assess liver disease in patients with hepatitis B or C. Gastroenterology 2012;142: 1293-302.
6. Udell JA, Wang CS, Tinmouth J, FitzGerald JM, Ayas NT, Simel DL, et al. Does this patient with liver disease have cirrhosis? J Am Med Assoc 2012;307:832-42.

7. Lin ZH, Xin YN, Dong QJ, Wang Q, Jiang XJ, Zhan SH, et al. Performance of the aspartate aminotransferase-to-platelet ratio index for the staging of hepatitis C-related fibrosis: an updated meta-analysis. Hepatology 2011;53:726-36.

8. Poynard T, Morra R, Halfon P, Castera L, Ratziu V, Imbert-Bismut F, et al. Meta-analyses of FibroTest diagnostic value in chronic liver disease. BMC Gastroenterol 2007;7:40.

9. Poynard T, Munteanu M, Deckmyn O, Ngo Y, Drane F, Messous D, et al. Applicability and precautions of use of liver injury 
biomarker FibroTest. A reappraisal at 7 years of age. BMC Gastroenterol 2011;11:39.

10. Degos F, Perez P, Roche B, Mahmoudi A, Asselineau J, Voitot $\mathrm{H}$, et al. Diagnostic accuracy of FibroScan and comparison to liver fibrosis biomarkers in chronic viral hepatitis: a multicenter prospective study (the FIBROSTIC study). J Hepatol 2010;53:1013-21.

11. Zarski JP, Sturm N, Guechot J, Paris A, Zafrani ES, Asselah T, et al. Comparison of nine blood tests and transient elastography for liver fibrosis in chronic hepatitis C: the ANRS HCEP-23 study. J Hepatol 2012;56:55-62.

12. Ghany MG, Kim HY, Stoddard A, Wright EC, Seeff LB, Lok AS, et al. Predicting clinical outcomes using baseline and follow-up laboratory data from the hepatitis C long-term treatment against cirrhosis trial. Hepatology 2011;54:1527-37.

13. Castéra L, Sebastiani G, Le Bail B, de Lédinghen V, Couzigou P, Alberti A. Prospective comparison of two algorithms combining non-invasive methods for staging liver fibrosis in chronic hepatitis C. J Hepatol 2010;52:191-8.

14. Boursier J, de Ledinghen V, Zarski JP, Fouchard-Hubert I, Gallois $\mathrm{Y}$, Oberti F, et al. Comparison of eight diagnostic algorithms for liver fibrosis in hepatitis $\mathrm{C}$ : new algorithms are more precise and entirely noninvasive. Hepatology 2012;55:58-67.

15. European Association for the Study of the Liver. EASL Clinical Practice Guidelines: management of hepatitis C virus infection. J Hepatol 2011;55:245-64.

16. Chalasani N, Younossi Z, Lavine JE, Diehl AM, Brunt EM, Cusi $\mathrm{K}$, et al. The diagnosis and management of non-alcoholic fatty liver disease: practice guideline by the American Gastroenterological Association, American Association for the Study of Liver Diseases, and American College of Gastroenterology. Gastroenterology 2012;142:1592-609. Erratum in: Gastroenterology 2012;143:503.

17. Targher G, Byrne CD. Nonalcoholic fatty liver disease: a novel cardiometabolic risk factor for type 2 diabetes and its complications. J Clin Endocrinol Metab 2013;98:483-95.

18. Bonora E, Targher G. Increased risk of cardiovascular disease and chronic kidney disease in NAFLD. Nat Rev Gastroenterol Hepatol 2012;9:372-81.

19. Williams KH, Shackel NA, Gorrell MD, McLennan SV, Twigg SM. Diabetes and nonalcoholic fatty liver disease: a pathogenic duo. Endocr Rev 2013;34:84-129.

20. Lee JY, Kim KM, Lee SG, Yu E, Lim YS, Lee HC, et al. Prevalence and risk factors of non-alcoholic fatty liver disease in potential living liver donors in Korea: a review of 589 consecutive liver biopsies in a single center. J Hepatol 2007;47:239-44.

21. Marcos A, Fisher RA, Ham JM, Olzinski AT, Shiffman ML, Sanyal AJ, et al. Selection and outcome of living donors for adult to adult right lobe transplantation. Transplantation 2000;69:2410-5.
22. Williams CD, Stengel J, Asike MI, Torres DM, Shaw J, Contreras $M$, et al. Prevalence of nonalcoholic fatty liver disease and nonalcoholic steatohepatitis among a largely middle-aged population utilizing ultrasound and liver biopsy: a prospective study. Gastroenterology 2011;140:124-31.

23. Browning JD, Szczepaniak LS, Dobbins R, Nuremberg P, Horton JD, Cohen JC, et al. Prevalence of hepatic steatosis in an urban population in the United States: impact of ethnicity. Hepatology 2004;40:1387-95.

24. Younossi ZM, Stepanova M, Negro F, Hallaji S, Younossi Y, Lam $B$, et al. Nonalcoholic fatty liver disease in lean individuals in the United States. Medicine (Baltimore) 2012;91:319-27.

25. Vajro P, Lenta S, Socha P, Dhawan A, McKiernan P, Baumann U, et al. Diagnosis of nonalcoholic fatty liver disease in children and adolescents: position paper of the ESPGHAN Hepatology Committee. J Pediatr Gastroenterol Nutr 2012;54:700-13.

26. Miller MH, Ferguson MA, Dillon JF. Systematic review of performance of non-invasive biomarkers in the evaluation of non-alcoholic fatty liver disease. Liver Int 2011;31:461-73.

27. Adams LA, Feldstein AE. Non-invasive diagnosis of nonalcoholic fatty liver and nonalcoholic steatohepatitis. J Dig Dis 2011;12:10-6.

28. Obika M, Noguchi H. Diagnosis and evaluation of nonalcoholic fatty liver disease. Exp Diabetes Res 2012;2012:145754.

29. Strnad P, Paschke S, Jang KH, Ku NO. Keratins: markers and modulators of liver disease. Curr Opin Gastroenterol 2012;28:209-16.

30. Shen J, Chan HL, Wong GL, Choi PC, Chan AW, Chan HY, et al. Non-invasive diagnosis of non-alcoholic steatohepatitis by combined serum biomarkers. J Hepatol 2012;56:1363-70.

31. Feldstein AE, Wieckowska A, Lopez AR, Liu YC, Zein NN, McCullough AJ. Cytokeratin-18 fragment levels as noninvasive biomarkers for nonalcoholic steatohepatitis: a multicenter validation study. Hepatology 2009;50:1072-8.

32. Joka D, Wahl K, Moeller S, Schlue J, Vaske B, Bahr MJ, et al. Prospective biopsy-controlled evaluation of cell death biomarkers for prediction of liver fibrosis and nonalcoholic steatohepatitis. Hepatology 2012;55:455-64.

33. Musso G, Gambino R, Cassader M, Pagano G. Meta-analysis: natural history of non-alcoholic fatty liver disease (NAFLD) and diagnostic accuracy of non-invasive tests for liver disease severity. Ann Med 2011;43:617-49.

34. Calès $P$, Lainé F, Boursier J, Deugnier $Y$, Moal V, Oberti F, et al. Comparison of blood tests for liver fibrosis specific or not to NAFLD. J Hepatol 2009;50:165-73.

35. Wong VW, Vergniol J, Wong GL, Foucher J, Chan HL, Le Bail B, et al. Diagnosis of fibrosis and cirrhosis using liver stiffness measurement in nonalcoholic fatty liver disease. Hepatology 2010;51:454-62. 